\title{
In vitro comparison of the hemocompatibility of diamond-like carbon and carbon nitride coatings with different atomic percentages of $\mathbf{N}$
}

\author{
ZHAO MengLi ${ }^{1}$, LI DeJun ${ }^{1 *}$, ZHANG YiTeng ${ }^{1}$, GUO MeiXian ${ }^{1}$, DENG XiangYun ${ }^{1}$, \\ GU HanQing ${ }^{2} \&$ WAN RongXin ${ }^{2}$ \\ ${ }^{1}$ College of Physics and Electronic Information Science, Tianjin Normal University, Tianjin 300387, China; \\ ${ }^{2}$ Tianjin Institute of Urological Surgery, Tianjin Medical University, Tianjin 300070, China
}

Received April 26, 2011; accepted February 6, 2012

\begin{abstract}
Carbon nitride $\left(\mathrm{CN}_{x}\right)$ and diamond-like carbon (DLC) coatings were prepared by dc magnetron sputtering at room temperature. Different partial pressures of $\mathrm{N}_{2}$ were used to synthesize $\mathrm{CN}_{x}$ to evaluate the relationship between the atomic percentage of nitrogen and hemocompatibility. Auger electron spectroscopy and atomic force microscopy indicated atomic percentages of $\mathrm{N}$ of 0.12 and 0.22 and that the $\mathrm{CN}_{x}$ coatings were smooth. An in vitro study of the hemocompatibility of the coatings revealed that both $\mathrm{CN}_{x}$ coatings had better anticoagulant properties and lower platelet adhesion than DLC. Compared with $\mathrm{CN}_{0.12}$, the $\mathrm{CN}_{0.22}$ coating showed longer dynamic clotting time (about $42 \mathrm{~min}$ ), static clotting time (23.6 min) and recalcification time (45.6 s), as well as lower platelet adhesion (102 cells $\mu \mathrm{m}^{-2}$ ), aggregation, and activation. The presence of nitrogen in the $\mathrm{CN}_{x}$ coatings induced their enhanced hemocompatibility compared with DLC.
\end{abstract}

carbon nitride, magnetron sputtering, atomic percentage, hemocompatibility

Citation: Zhao M L, Li D J, Zhang Y T, et al. In vitro comparison of the hemocompatibility of diamond-like carbon and carbon nitride coatings with different atomic percentages of N. Sci China Life Sci, 2012, 55: 343-348, doi: 10.1007/s11427-012-4308-9

The development of new materials that can be used as medical implants has received much interest in recent years. Implants can be in contact with inorganic materials, biomacromolecules, cells, tissues and organs in the human body, so they must be fabricated from appropriate materials with controlled properties. In the selection of a synthetic material for use in biological media, there are two important considerations, namely, (i) the compatibility of a material with the biological environment, and (ii) the specific properties of a material that can be varied following implantation. For the first requirement, a material must not induce an adverse response such as excessive deposition of fluid components, abnormal cell growth, toxic or allergic reac-

*Corresponding author (email: dejunli@mail.tjnu.edu.cn) tions, or thrombosis in the case of blood-contacting devices. For the second requirement, the material should possess satisfactory resistance to tension, shear, wear, and corrosion depending on the specific application [1].

Carbon films are known for their broad structural variability and a correspondingly wide range of properties varying from diamond- to graphite-like. This variability is determined by the ratio of diamond bonds $\left(\mathrm{sp}^{3}\right)$ to graphitic bonds $\left(\mathrm{sp}^{2}\right)$ in the sample [2]. The biocompatibility and low wettability of diamond-like carbon (DLC) [3] means that it shows potential for application in biology and medicine as a protective coating for implants, biosensors and biochips [2,4-7]. It is anticipated that amorphous DLC will be used in medical devices such as hip prostheses, coronary stents, heart valves, vascular prostheses, intraocular and contact 
lenses, surgical needles for corneal surgery, orthopedic pins and screws, dental prostheses, medical guidewires, and rotary pumps for left ventricular assistance [7-15].

Carbon nitride $\left(\mathrm{CN}_{x}\right)$ coatings containing a mixture of $\mathrm{sp}^{2}$ and $\mathrm{sp}^{3}$ bonds are structurally similar to DLC. The hardness and wear resistance of $\mathrm{CN}_{x}$ coatings are similar to those of DLC $[16,17]$. Despite the promise of DLC in biomedical applications, the similar structural, mechanical and tribological properties of $\mathrm{CN}_{x}$ and DLC, and the probable improved biocompatibility because of the presence of nitrogen, $\mathrm{CN}_{x}$ has not received attention as a coating for implants and its hemocompatibility has not been assessed [18]. $\mathrm{CN}_{x}$ coatings consist mainly of carbon and nitrogen and some may be doped with hydrogen. Because these elements are widely spread in the human body, $\mathrm{CN}_{x}$ may possess excellent biocompatibility. In our previous study [19], magnetron sputtering was employed to fabricate smooth $\mathrm{CN}_{x}$ coatings with acceptable corrosion performance. In this work, our aim was to prepare $\mathrm{CN}_{x}$ coatings using standard magnetron sputtering, and study the effects of the atomic concentration of $\mathrm{N}$ on blood-clotting time as well as platelet adhesion to the coatings.

\section{Materials and methods}

\subsection{Preparation of DLC and $\mathrm{CN}_{x}$ coatings}

$\mathrm{CN}_{x}$ and DLC coatings were prepared by dc magnetron sputtering at room temperature. The base pressure in the chamber was below $6 \times 10^{-6} \mathrm{~Pa}$. Graphite (purity $99.995 \%$ ) was used as the target. DLC coatings were prepared using $\mathrm{Ar}$ as the process gas. An $\mathrm{Ar}-\mathrm{N}_{2}$ mixture was used as the process gas to prepare $\mathrm{CN}_{x}$ coatings. During deposition, the total pressure was kept at 1.5 mTorr, and the target power was fixed at $150 \mathrm{~W}$. Two different partial pressures of $\mathrm{N}_{2}$ (0.1 and 0.5 mTorr) were used to synthesize $\mathrm{CN}_{x}$ coatings containing different atomic percentages of N. A constant dc bias of $-80 \mathrm{~V}$ was applied to the substrates during deposition. Silicon (100) wafers, which were cleaned ultrasonically in acetone and methanol for $10 \mathrm{~min}$ before loading into the chamber, were used as substrates.

\subsection{Characterization of coatings}

Auger electron spectroscopy (AES) was used to determine the chemical composition and atomic percentage of $\mathrm{N}$ in the coatings. The chemical bonding states of the DLC coating were characterized by Raman spectroscopy using a Renishaw micro-Raman 2000 spectrometer (Renishaw, Britain) and $514 \mathrm{~nm}$ laser excitation. The root-mean-square (RMS) surface roughness was obtained for sample areas ranging from 1 to $10 \mu \mathrm{m}^{2}$ using atomic force microscopy (AFM). The contact angles of water on $\mathrm{CN}_{x}$ and DLC coatings as well as the control $\mathrm{Si}$ surfaces were measured at room temperature using a CAM 100 Contact Angle Instrument (KSV,
Finland). Measurements were repeated in three different areas on each surface.

\subsection{Platelet adhesion, and dynamic and static clotting time assays}

Samples that were uncoated and coated with $\mathrm{CN}_{x}$ or DLC with a thickness of $50 \mathrm{~nm}$ were sterilized in ethylene oxide prior to use in hemocompatibility measurements. Dynamic clotting time was measured by the dynamic method. In this method, blood $(0.1 \mathrm{~mL})$ from a healthy adult volunteer was immediately added dropwise onto the surface of each sample. After a predetermined time, the samples were transferred into a beaker containing distilled water $(50 \mathrm{~mL})$. The red blood cells that had not been trapped in a thrombus were hemolyzed, and the free hemoglobin was dispersed in the solution. The concentration of free hemoglobin in the solution was determined colorimetrically at $540 \mathrm{~nm}$ with a spectrophotometer. The optical density at $540 \mathrm{~nm}\left(A_{540 \mathrm{~nm}}\right)$ of the solution vs. time was plotted. In general, $A_{540 \mathrm{~nm}}$ decreases with increased blood clotting.

The static clotting time of the coatings was investigated using rabbit blood $(1 \mathrm{~mL})$ left in contact with the coated surface at $37^{\circ} \mathrm{C}$ using a orbital shaker water bath. The recalcification time of the coatings was measured by recording the interval of time from introducing of $\mathrm{CaCl}_{2}$ solution $(0.1$ $\mathrm{mL})$ into a test-tube containing the sample and rabbit plas$\mathrm{ma}(0.1 \mathrm{~mL})$ to observing white particles in the plasma.

The number and morphology of adhered platelets were measured to determine the hemocompatibility of the surfaces. Blood $(60 \mathrm{~mL})$ drawn from a healthy adult volunteer who free of aspirin or other drugs that might interfere with platelet function was centrifuged for about 10 min to obtain platelet-rich plasma. The platelet-rich plasma was added dropwise onto the surfaces, and incubated at $37^{\circ} \mathrm{C}$ for 15 min. After rinsing, fixing and critical point drying, the surfaces were coated with gold-palladium, and observed by optical microscopy and scanning electron microscopy (SEM). Twenty $1 \mathrm{~mm}^{2}$ fields of vision were investigated to determine the typical morphology and statistical results of platelet adhesion.

\section{Results}

\subsection{Characterization by AES, Raman and AFM}

AES shown in Figure 1 reveal the presence of mainly carbon and nitrogen in the coatings prepared under different partial pressures of $\mathrm{N}_{2}$. The oxygen signal (atomic percentage of $\sim 5 \%$ ) is caused by exposure of the sample to air during transport and is readily removed by argon sputtering for $3 \mathrm{~min}$. The N/C ratio is obtained by converting auger intensities into atomic percentages. In this calculation, the sensitivity factors are 0.2 for carbon and 0.3 for nitrogen. At a total pressure of 1.5 mTorr, a partial pressure of $\mathrm{N}_{2}$ of 0.1 
mTorr gives an atomic percentage of $\mathrm{N}$ of $12 \%$. This value increases to $22 \%$ for the coating prepared under a partial pressure of $\mathrm{N}_{2}$ of 0.5 mTorr. A Raman spectrum of the DLC coating showed typical D-mode $\left((1334.3 \pm 3.5) \mathrm{cm}^{-1}\right)$ and G-mode $\left((1531.8 \pm 0.9) \mathrm{cm}^{-1}\right)$ vibration bands. The half widths of the D- and G-modes obtained from Gaussian fitting were $(345.51 \pm 13.6)$ and $(206.96 \pm 2.7) \mathrm{cm}^{-1}$, respectively. The ratio of peak areas, $I_{\mathrm{D}} / I_{\mathrm{G}}$, was 1.469 .

The RMS roughness of DLC, $\mathrm{CN}_{0.12}$, and $\mathrm{CN}_{0.22}$ coatings

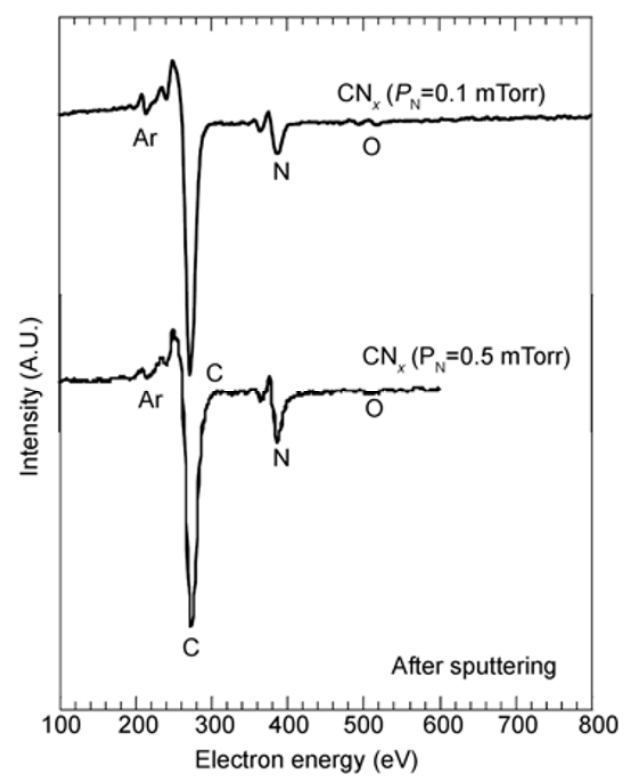

Figure 1 AES of $\mathrm{CN}_{x}$ coatings formed under different partial pressures of $\mathrm{N}_{2}$. as a function of AFM scan length is shown in Figure 2. AFM images of DLC and $\mathrm{CN}_{0.22}$ coatings with sample areas of $10 \mu \mathrm{m}^{2}$ are also displayed in this figure. $\mathrm{CN}_{x}$ coatings formed under the same experimental conditions as DLC coatings were slightly smoother using a scan length of 10 $\mu \mathrm{m}$. In addition, the surface of the $\mathrm{CN}_{x}$ coating became smoother at higher nitrogen content. The addition of nitrogen causes the atoms to bond tightly, which induces formation of a dense structure with a smooth surface.

\subsection{Water contact angles of coatings}

In actual practice, instruments do not directly measure the surface free energy of solid materials. Therefore, the water contact angles measured on the surfaces of samples are used to qualitatively analyze surface free energy. Nitrogen has a significant influence on water contact angle. Generally speaking, the surface free energy of a sample increases when the contact angle between it and water decreases, provided the contact angle is less than $90^{\circ}$ [20]. The water contact angles obtained for DLC, $\mathrm{CN}_{0.12}$ and $\mathrm{CN}_{0.22}$ coatings were $84.1^{\circ}, 73.2^{\circ}$, and $57.9^{\circ}$, respectively. The contact angles of the $\mathrm{CN}_{x}$ coatings are smaller than that of DLC, indicating that doping with nitrogen generates a hydrophilic surface.

\subsection{Dynamic clotting time}

The relationships between the optical density at $540 \mathrm{~nm}$ $\left(A_{540 \mathrm{~nm}}\right)$ of the free hemoglobin in healthy human blood solution and the blood clotting on the surfaces of $\mathrm{CN}_{x}$ and

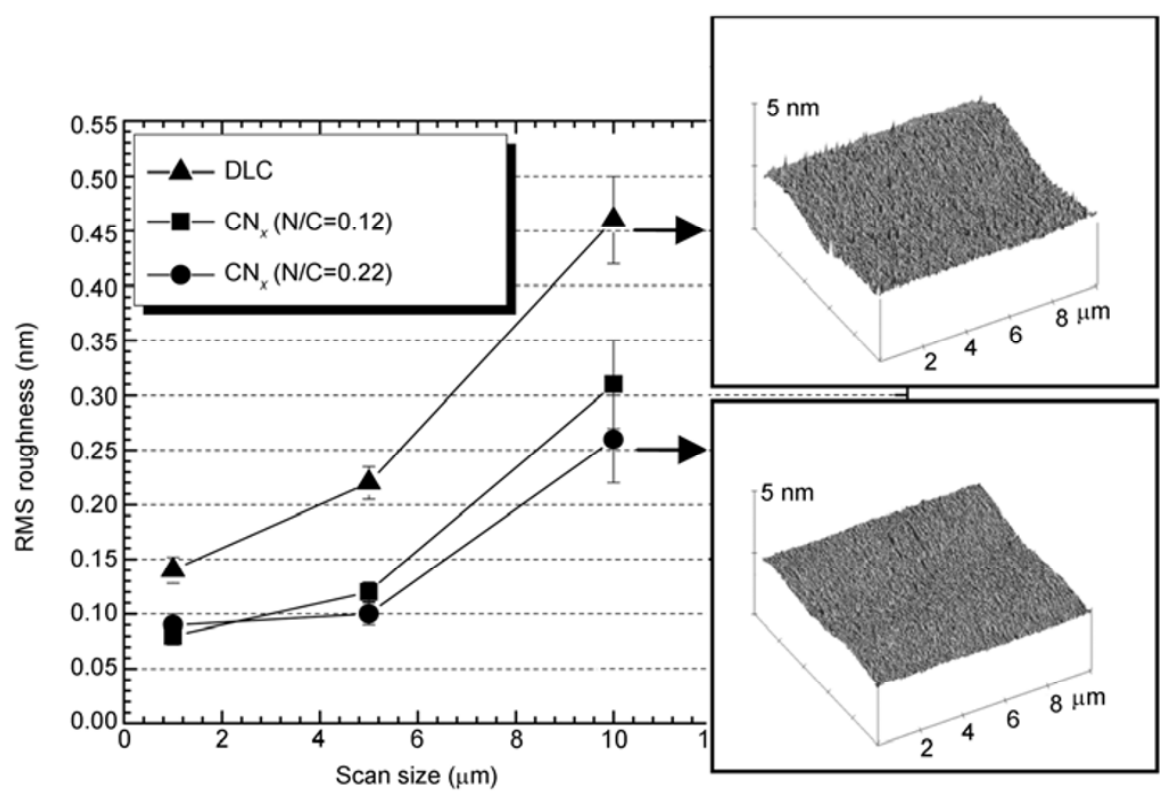

Figure 2 Variation of the RMS roughness as a function of AFM scan length for DLC and $\mathrm{CN}_{x}$ coatings. AFM images of the DLC (top) and CN 0.22 (bottom) coatings are presented in the insets. 
DLC coatings, and the control Si substrate are presented in Figure 3 . The dynamic clotting time of the coatings are also shown. Each value in this figure represents the average of five measurements. When the blood on the sample surfaces was completely clotted, $A_{540}$ of the solution decreased to a stable value. In general, blood starts to clot when $A_{540}=0.1$, at which point the dynamic clotting time was recorded. The dynamic clotting time on DLC coating is $262 \%$ longer than that on $\mathrm{Si}$, suggesting that is a better anticoagulant that the control Si substrate. The longest dynamic clotting time is observed for the $\mathrm{CN}_{0.22}$ coating (42 $\mathrm{min}$ ), and is more than twice as long as that for DLC (29 min), which implies that the $\mathrm{CN}_{0.22}$ coating has good hemocompatibility.

\subsection{Static clotting time}

Static clotting time and recalcification times for the surfaces were determined using venous blood from a rabbit (Figure 4). Longer static clotting and recalcification times indicate better anticoagulant properties. Values in this figure represent the average of ten measurements. Significant differences between the DLC, $\mathrm{CN}_{0.12}, \mathrm{CN}_{0.22}$, and control Si coatings were observed. The static clotting and recalcification times of DLC coating are almost $25 \%$ longer than those of the control Si substrate, and $17.9 \%$ shorter than those of the $\mathrm{CN}_{0.12}$ coating. The $\mathrm{CN}_{0.22}$ coating exhibited the longest static clotting and recalcification times of the coatings, which is agreement with the dynamic clotting times.

\subsection{Platelet adhesion test}

SEM images of platelets adhered to DLC, $\mathrm{CN}_{x}$ and control Si surfaces after an incubation time of $15 \mathrm{~min}$ are shown in Figure 5. The numbers of platelets on the surfaces were also counted (Figure 6). Values in Figure 6 represent the average of five measurements. It is clear that the number of individ- ual platelets on the DLC and $\mathrm{CN}_{x}$ coatings is lower than that on the control Si. A large amount of activated platelets with a certain degree of spreading are observed on the control $\mathrm{Si}$ and DLC coating. Many deformed platelets, such as pseudopodia, are also observed on the surface of Si and DLC coating, as shown in Figure 5A and B, respectively. However, the platelets adhered to $\mathrm{CN}_{x}$ surfaces are present in lower number, typically $2-5 \mu \mathrm{m}$ in size and are disc-shaped, although some platelets exhibit the early stages of activation (Figure 5C and D). Compared with DLC and $\mathrm{CN}_{0.12}$ coatings, the $\mathrm{CN}_{0.22}$ coating shows the lowest platelet aggregation and activation because few pseudopodia are present. The number of adhered platelets is lowest for the $\mathrm{CN}_{0.22}$ coating (102 cells $\mu \mathrm{m}^{-2}$, Figure 6 ). This implies that the $\mathrm{CN}_{0.22}$ coating inhibits aggregation of platelets and the formation of pseudopodium, providing further evidence for its hemocompatibility.

\section{Discussion}

Thrombogenicity occurring on the surface of foreign materials while in contact with blood is a very complicated process and is far from being well understood. Factors influencing the hemocompatibility of biomaterials include surface roughness, hydrophilicity or hydrophobicity, and surface charge density. It is well known that strong adhesion and aggregation of platelets on a surface are related to rapid blood-clotting. A smooth surface improves the hemocompatibility of biomaterials because it inhibits platelet agglutination and fibrin thrombus formation induced by thrombin on the sample surface. The $\mathrm{CN}_{0.22}$ coating possesses the smoothest surface of those investigated, as shown in Figure 2, which may have a positive effect on biocompatibility.

In addition, many studies have demonstrated that higher hydrophilicity and surface energy appeared to be the pri-

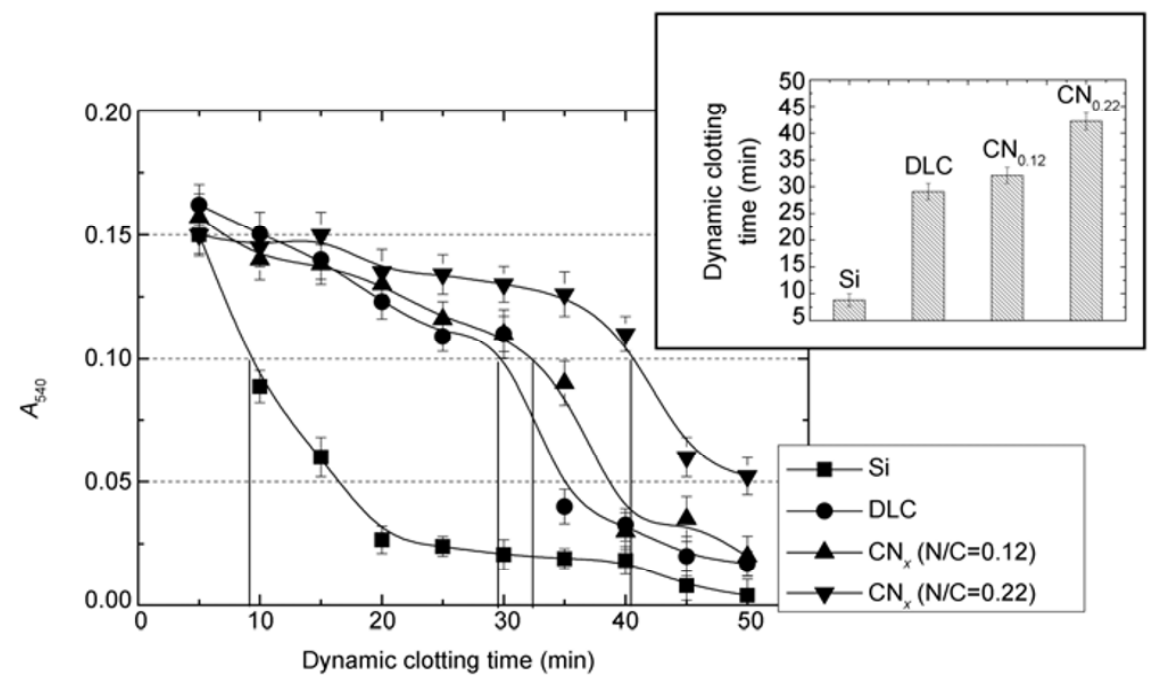

Figure $3 A_{540}$ obtained for $\mathrm{Si}$, DLC, and $\mathrm{CN}_{x}$ coatings vs. blood-clotting time. Dynamic clotting times are shown in the inset. 


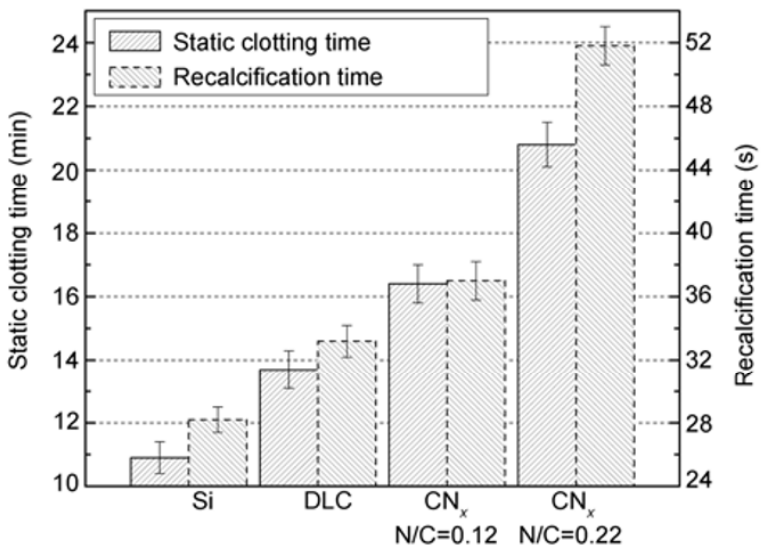

Figure 4 Static clotting and recalcification times for $\mathrm{Si}$, DLC, and $\mathrm{CN}_{x}$ coatings.

mary factors improving surface blood-contact properties, and can significantly reduce platelet adhesion and aggregation [21,22]. Materials with high hydrophilicity and surface energy are likely to be covered by a protein-dominating 'conditioning film' that may yield good hemocompatibility [23,24]. In this study, the contact angles of the $\mathrm{CN}_{0.22}$ coating is the lowest, so it also possesses the highest surface energy of the surfaces. A high atomic percentage of $\mathrm{N}$ should have a significant effect on hydrophilicity and surface energy, explaining why the $\mathrm{CN}_{0.22}$ coating exhibits the best hemocompatibility.

Baurschmidt and Schaldach [25] reported that the formation of thrombus on the surface of a biomaterial is correlated with charge transfer from fibrinogen to the material surface. Fibrinogen can transform into fibrin monomers and fibrinopeptides when it loses charge. The crosslinking of fibrin monomers causes an irreversible thrombus. Thus, a suitable density of charge will improve hemocompatibility [26]. The substitution of nitrogen for carbon induces a variety of new bonding configurations because nitrogen has five valence electrons, although despite this extra valence electron, nitrogen forms similar hybridized states to carbon. In $\mathrm{sp}^{3}$ hybridization, the extra valence electron fills one of the four $\mathrm{sp}^{3}$ orbitals, resulting in three $\mathrm{sp}^{3}$ orbitals with one electron each, which can be used to form a trigonal pyramid, and the other $\mathrm{sp}^{3}$ orbital is nonbonding, containing two electrons in a lone pair [27-29]. The lone pair of electrons prevented charge transfer from fibrinogen to the material surface, which improved the hemocompatibility of the $\mathrm{CN}_{x}$ coatings.
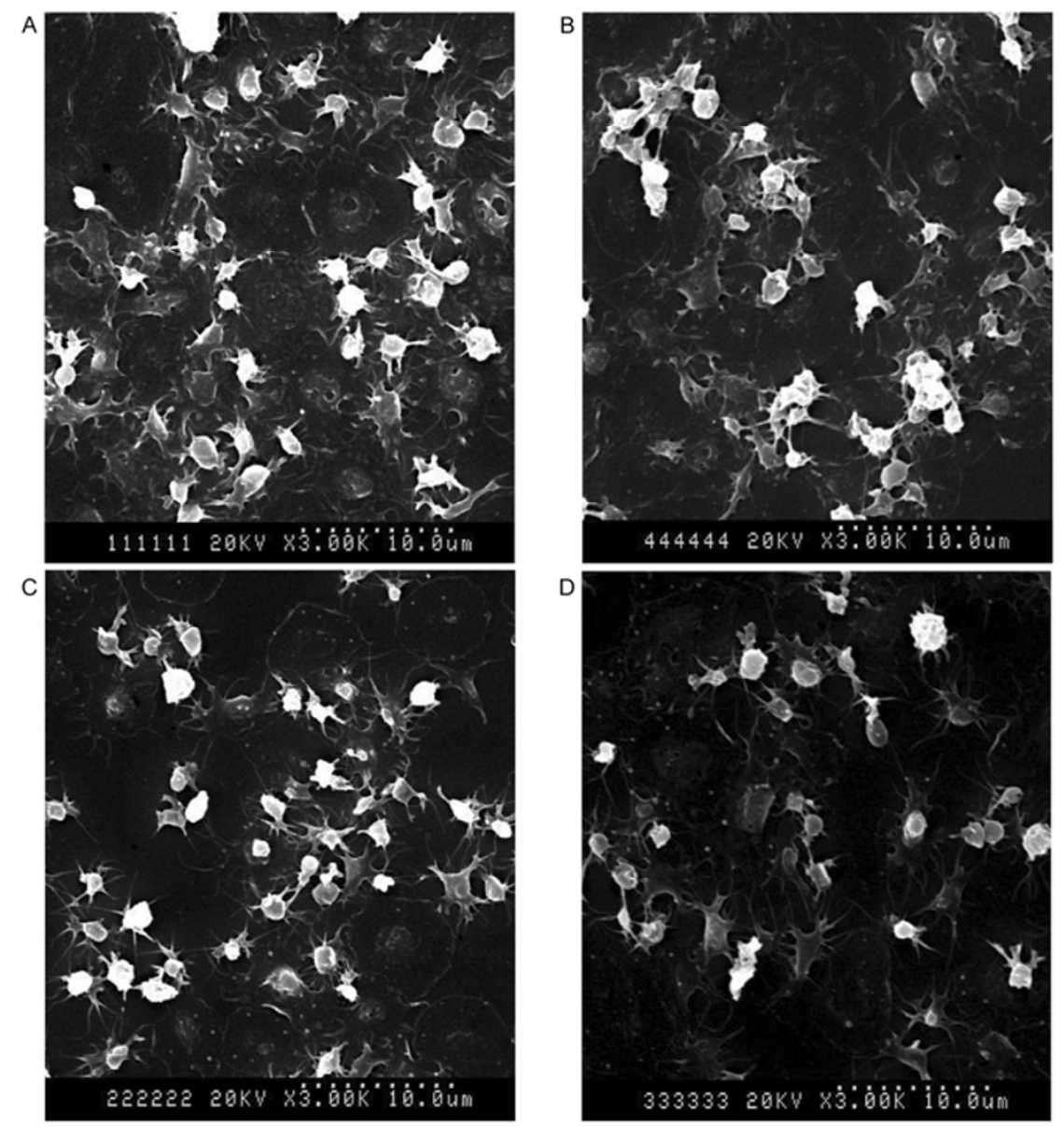

Figure 5 SEM images of platelets on $\mathrm{Si}(\mathrm{A}), \mathrm{DLC}(\mathrm{B}), \mathrm{CN}_{0.12}(\mathrm{C})$ and $\mathrm{CN}_{0.22}$ (D) surfaces. 


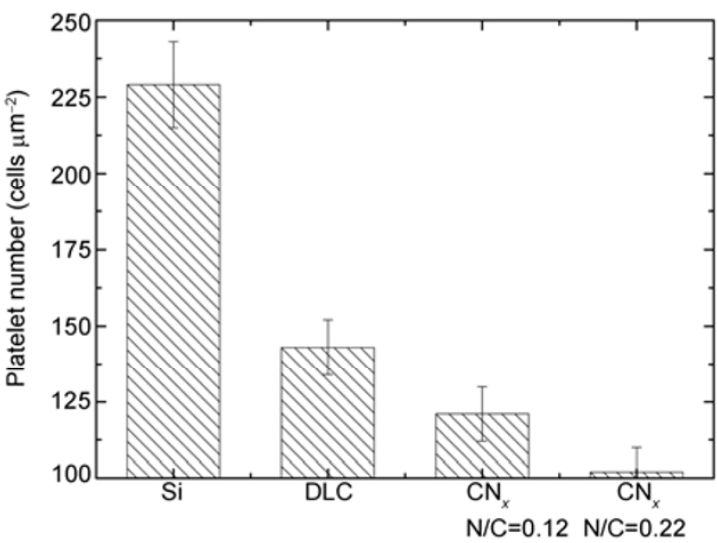

Figure 6 Number of platelets adhered to $\mathrm{Si}$, DLC, and $\mathrm{CN}_{x}$ surfaces.

\section{Conclusion}

$\mathrm{CN}_{x}$ coatings were prepared using two partial pressures of $\mathrm{N}_{2}$ at room temperature by standard magnetron sputtering. A higher atomic percentage of $\mathrm{N}$ can be introduced into the coating under a higher partial pressure of $\mathrm{N}_{2} \cdot \mathrm{CN}_{x}$ coatings were shown to exhibit longer dynamic and static clotting and recalcification times, and lower platelet adhesion, aggregation and platelet activation than a DLC coating formed under the same conditions. The $\mathrm{CN}_{x}$ coating showed an observable improvement in hemocompatibility as the atomic percentage of $\mathrm{N}$ was increased. These results should initiate an interest in the biomedical applications of $\mathrm{CN}_{x}$ coatings.

This work was supported by the National Basic Research Program of China (Grant No. 2012CB933604), the National Natural Science Foundation of China (Grant No. 11075116), the National Natural Science Foundation of China "The effect of functionalization induced by ion beam on cell and blood compatibility of graphene" (2013), the Open Research Fund of the State Key Laboratory of Bioelectronics, Southeast University, and the Key Laboratory of Beam Technology and Material Modification of Ministry of Education, Beijing Normal University, China.

1 Ruckenstein E, Gourisankar S V. Preparation and characterization of thin film surface coatings for biological environments. Biomaterials, 1986, 7: 403-422

2 Jelinek M, Smetana K, Kocoureka T, et al. Biocompatibility and $\mathrm{sp}^{3} / \mathrm{sp}^{2}$ ratio of laser created DLC films. Mater Sci Eng B, 2010, 169: 89-93

3 Yang P, Huang N, Leng Y X, et al. Activation of platelets adhered on amorphous hydrogenated carbon $(\mathrm{a}-\mathrm{C}: \mathrm{H})$ films synthesized by plasma immersion ion implantation-deposition (PIII-D). Biomaterials, 2003, 24: 2821-2829

4 Salgueiredo E, Vila M, Silva M A, et al. Biocompatibility evaluation of DLC-coated $\mathrm{Si}_{3} \mathrm{~N}_{4}$ substrates for biomedical applications. Dia Rel Mater, 2008, 17: 878-881

5 Bharathy P V, Nataraj D, Chu P K, et al. Effect of titanium incorporation on the structural, mechanical and biocompatible properties of DLC thin films prepared by reactive-biased target ion beam deposition method. Appl Surf Sci, 2010, 257: 143-150

6 Pandiyaraj K N, Selvarajan V, Heeg J, et al. Influence of bias voltage on diamond like carbon (DLC) film deposited on polyethylene ter- ephthalate (PET) film surfaces using PECVD and its blood compatibility. Dia Rel Mater, 2010, 19: 1085-1092

7 Dearnaley G, Arps J H. Biomedical applications of diamond-like carbon (DLC) coatings: A review. Surf Coat Technol, 2005, 200: 2518-2524

8 Maa W J, Ruysa A J, Masonb R S, et al. Use of diamond-like carbon with tungsten (W-DLC) films as biocompatible material. Biomaterials, 2007, 28: 1620-1628

9 Lousinian S, Logothetidis S, Laskarakis A, et al. Haemocompatibility of amorphous hydrogenated carbon thin films, optical properties and adsorption mechanisms of blood plasma proteins. Biomol Eng, 2007, 24: $107-112$

10 Uzumaki E T, Lambert C S, Santos A R, et al. Surface properties and cell behaviour of diamond-like carbon coatings produced by plasma immersion. Thin Solid Films, 2006, 515: 293-300

11 Allen M, Myer B, Rushton N. In vitro and in vivo investigations into the biocompatibility of diamond-like carbon (DLC) coatings for orthopedic applications. J Biomed Mater Res, 2001, 58: 319-328

12 Cui F Z, Qing X L, Li D J, et al. Biomedical investigations on $\mathrm{CN}_{x}$ coating. Surf Coat Technol, 2005, 200: 1009-1013

13 Jones M I, McColl I R, Grant D M, et al. Structure and properties of annealed amorphous hydrogenated carbon (a-C:H) films for biomedical applications. Dia Rel Mater, 1999, 8: 457-462

14 Bendavid A, Martin P J, Comte C, et al. The mechanical and biocompatibility properties of DLC-Si films prepared by pulsed DC plasma activated chemical vapor deposition. Dia Rel Mater, 2007, 16: 1616-1622

15 Marton M, Zdravecka E, Vojs M, et al. Study of adhesion of carbon nitride thin films on medical alloy substrates. Vacuum, 2010, 84: 65-67

16 Rossi F, Andre B, Van Veen A, et al. Physical properties of nitrogenated amorphous carbon films produced by ion-beam-assisted deposition. Thin Solid Films, 1994, 253: 85-89

17 Yu L J, Wang X, Wang X H, et al. Haemocompatibility of tetrahedral amorphous carbon films. Surf Coat Technol, 2000, 128-129: 484-488

18 Zheng C L, Cui F Z, Meng B, et al. Hemocompatibility of C-N films fabricated by ion beam assisted deposition. Surf Coat Technol, 2005, 193: $361-365$

19 Li D J, Guruz M U, Bhatia C S, et al. Ultrathin $\mathrm{CN}_{x}$ overcoats for 1 Tb/in. ${ }^{2}$ hard disk drive systems. Appl Phys Lett, 2002, 81: 1113-1115

20 Milojka G, Gerhard S, Wolfgang G, et al. A comparison of different methods to calculate the surface free energy of wood using contact angle measurements. Colloids Surf A Physicochem Eng Asp, 2001, 181: 279-287

21 Dynes P J, Kaelble D H. Surface energy analysis of carbon fibers and films. J Adhes, 1974, 6: 195-199

22 Laura J S, Jennifer L W, Antonios G M. Platelet adhesion on a bioresorbable poly(propylene fumarate-co-ethylene glycol) copolymer. Biomaterials, 1999, 20: 683-690

23 Ivarsson B, Lundstrom I. Physical characterization of protein adsorption on metal and metaloxide surfaces. CRC Crit Rev Biocompat, 1986, 2: 1-96

24 Baier R E. Conditioning surfaces to suit the biomedical environment. J Biomech Eng, 1982, 104: 257-260

25 Baurschmidt P, Schaldach M. Alloplastic materials for heart-valve prostheses. Med Biol Eng Comput, 1980, 18: 496-502

26 Zhang L, Chen M, Li Z Y, et al. Effect of annealing on structure and haemocompatibility of tetrahedral amorphous hydrogenated carbon films. Mater Lett, 2008, 62: 1040-1043

27 Titantah J T, Lamoen D. Carbon and nitrogen 1s energy levels in amorphous carbon nitride systems: XPS interpretation using first-principles. Dia Rel Mater, 2007, 16: 581-588

28 Hultman L, Neidhardt J, Hellgren N, et al. Fullerene-like carbon nitride: A resilient coating material. MRS Bull, 2003, 28: 194-202

29 Ferrari A C, Kleinsorge B, Morrison N A, et al. Stress reduction and bond stability during thermal annealing of tetrahedral amorphous carbon. J Appl Phys, 1999, 85: 7191-7197

Open Access This article is distributed under the terms of the Creative Commons Attribution License which permits any use, distribution, and reproduction in any medium, provided the original author(s) and source are credited. 